\title{
Experimental acute pancreatitis in PAP/HIP knock-out mice
}

\author{
Meritxell Gironella, Emma Folch-Puy, Aude LeGoffic, Stéphane Garcia, Laurence Christa, Andrew \\ Smith, Luis Tebar, Stephen P Hunt, Rosemary Bayne, Andrew J H Smith, Jean-Charles Dagorn, Daniel \\ Closa, Juan L lovanna
}

Gut 2007;56:1091-1097. doi: 10.1136/gut.2006.116087

See end of article for authors' affiliations

Correspondence to:

Dr J L lovanna, Centre de Recherche INSERM, Unité 624, Stress Cellulaire, 163 Avenue de Luminy, BP915, 13288 Marseille, France; iovanna@marseille.inserm.fr

Revised 13 March 2007 Accepted 20 March 2007 Published Online First 4 April 2007
Background and aims: PAP/HIP was first reported as an additional pancreatic secretory protein expressed during the acute phase of pancreatitis. It was shown in vitro to be anti-apoptotic and anti-inflammatory. This study aims to look at whether PAP/HIP plays the same role in vivo.

Methods: A model of caerulein-induced pancreatitis was used to compare the outcome of pancreatitis in $\mathrm{PAP} / \mathrm{HIP}^{-/-}$and wild-type mice.

Results: PAP/HIP ${ }^{-1-}$ mice showed the normal phenotype at birth and normal postnatal development. Caerulein-induced pancreatic necrosis was, however, less severe in PAP/HIP ${ }^{-/}$mice than in wild-type mice, as judged by lower amylasemia and lipasemia levels and smaller areas of necrosis. On the contrary, pancreas from PAP/HIP ${ }^{-/-}$mice was more sensitive to apoptosis, in agreement with the anti-apoptotic effect of PAP/HIP in vitro. Surprisingly, pancreatic inflammation was more extensive in PAP/HIP ${ }^{-1-}$ mice, as judged from histological parameters, increased myeloperoxidase activity and increased pro-inflammatory cytokine expression. This result, in apparent contradiction with the limited necrosis observed in these mice, is, however, in agreement with the anti-inflammatory function previously reported in vitro for PAP/HIP. This is supported by the observation that activation of the STAT3/SOCS3 pathway was strongly decreased in the pancreas of PAP/HIP-/- mice and by the reversion of the apoptotic and inflammatory phenotypes upon administration of recombinant PAP/HIP to PAP/HIP ${ }^{-/}$mice.

Conclusion: The anti-apoptotic and anti-inflammatory functions described in vitro for PAP/HIP have physiological relevance in the pancreas in vivo during caerulein-induced pancreatitis.
T he pancreatitis-associated protein (PAP) was first reported by Keim and co-workers ${ }^{1}$ in 1984 as an additional pancreatic secretory protein expressed during acute pancreatitis. It accounts for approximately $5 \%$ of the protein secreted during the acute phase and returns to almost undetectable levels when the pancreas has totally recovered. ${ }^{2}$ Although PAP was originally characterized in the pancreas, its expression was also observed in a variety of tissues, including epithelial cells of the small intestine, ${ }^{3}$ pituitary gland, ${ }^{4}$ uterus ${ }^{5}$ and motoneurons. ${ }^{6}$ PAP expression is upregulated in diseases such as Crohn's disease and ulcerative colitis, ${ }^{7}$ colorectal cancer, ${ }^{8}$ hepatocellular cancer and cholangiocarcinoma. ${ }^{9}{ }^{10}$ The primary structure of PAP was determined after cloning the corresponding messenger RNA from rat, ${ }^{11}$ mouse, in which it was called RegIII $\beta^{12}$ and human pancreas. ${ }^{13}$ Concomitantly, Lasserre and colleagues $^{10}$ found the same transcript overexpressed in several hepatocellular carcinomas and named the encoded protein HIP. Therefore, hereafter we will call this gene PAP/HIP.

$\mathrm{PAP} / \mathrm{HIP}$ expression is induced in pancreatic acinar cells in response to several cytokines such as tumour necrosis factor $\alpha$ $(\mathrm{TNF} \alpha)$, interferon $\gamma$, interleukin (IL) 6 in combination with dexamethasone, ${ }^{14}$ oxidative stress inducers such as hydrogen peroxide or menadione ${ }^{15}$ and lipopolysaccharide. ${ }^{16}$ In addition to the pro-inflammatory factors described above, IL10 ${ }^{17}$ and the IL10-related cytokine IL22 ${ }^{18}$ mediate in vitro and in vivo robust induction of PAP/HIP mRNA in pancreatic acinar cells. Finally, $\mathrm{PAP} / \mathrm{HIP}$ is able to induce its own expression through a STAT3mediated pathway creating a positive feedback. ${ }^{17}$

The physiological role of PAP/HIP remains unclear, although several functions have been suggested. Data support its involvement in tissue regeneration and cell proliferation, ${ }^{6}{ }_{19} 20$ whereas overexpression of PAP/HIP also increases resistance to apoptosis induced by oxidative stress ${ }^{15}$ or $\mathrm{TNF}^{21}$ in pancreatic acinar cells, motoneurons ${ }^{22}$ and hepatocytes. ${ }^{20}{ }^{23}$ Interestingly, several works also suggest that PAP/HIP could be an antiinflammatory factor. ${ }^{74-26}$ An anti-inflammatory activity of PAP/HIP would be in agreement with its strong induction observed during the course of inflammatory diseases such as pancreatitis, Crohn's disease and ulcerative colitis. In fact, recent data have shown that PAP/HIP is able to activate Jak kinase, which in turn phosphorylates STAT3, resulting in its nuclear translocation. ${ }^{17}$ Activated STAT3 triggers the expression of the suppressor of cytokine signaling 3 (SOCS3) gene. Once synthesized, SOCS3 binds the regulatory tyrosine located to the activation loop of Jak, blocking the access to the active site and preventing further phosphorylation. Therefore, the negative feedback mediated by SOCS3, which inhibits activation of the Jak/STAT3 cascade, seems to be a key regulatory element of the PAP/HIP anti-inflammatory pathway.

Evidence that the stress protein PAP/HIP can be mitogenic, anti-apoptotic and anti-inflammatory, three functions involving distinct molecular mechanisms, implies that it could coordinate defence-repair activities upon cell injury. As a first step to test that hypothesis, we used a PAP/HIP-deficient mouse model to obtain a deeper insight into the role of PAP/HIP in the exocrine pancreas during experimental pancreatitis.

\section{MATERIALS AND METHODS}

\section{Induction of experimental pancreatitis}

We used PAP/HIP ${ }^{-1-}$ mice generated as previously reported. ${ }^{27}$ Pancreatitis was induced in 4-month-old PAP/HIP ${ }^{-/-}$and PAP/

Abbreviations: IL, interleukin; PAP, ; pancreatitis-associated protein, ; PARP, poly(adenosine diphosphate-ribose) polymerase; PMN, polymorphonuclear; RT-PCR, reverse transcriptase-polymerase chain reaction; TBS, Tris-buffered saline; TNF $\alpha$, tumour necrosis factor $\alpha$ 
A

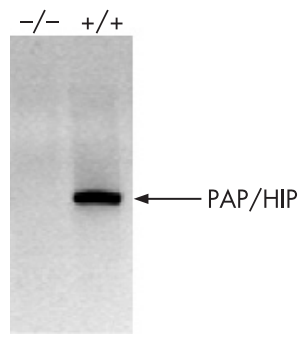

B

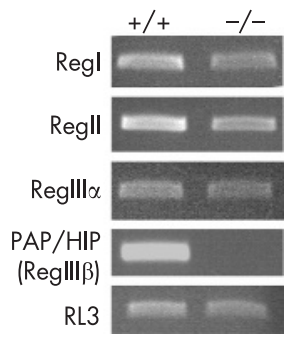

Figure 1 Expression of PAP/HIP family members in the pancreas of PAP/ $\mathrm{HIP}^{+/+}$and PAP/HIP ${ }^{-/-}$mice. A. Expression of PAP/HIP was analyzed by Western blotting, using a specific polyclonal antibody, in the pancreas of $\mathrm{PAP} / \mathrm{HIP}^{+/+}$and PAP/HIP ${ }^{-1-}$ mice during acute pancreatitis. Expression is restricted to PAP/HIP ${ }^{+/+}$mice, as expected. B. Expression of PAP/HIP family members in the pancreas of PAP/HIP $\mathrm{H}^{+/+}$and $\mathrm{PAP} / \mathrm{HIP}^{-/-}$mice. Assessment of mRNA levels of Regl, Regll, Reglll $\alpha$ and PAP/HIP (Reglll $\beta$ ) by semiquantitative reverse transcriptase-polymerase chain reaction analysis of pancreatic RNA from PAP/HIP $\mathrm{HI}^{+/+}$and PAP/HIP $/ /-$mice, 9 hours after caerulein treatment. RL3 mRNA was used as a housekeeping control. Results from one representative mouse for each group are included. Reglll $\gamma$ could not be evidenced in PAP/HIP $+/+$, although the same primers could detect a transcript in intestinal RNA (not shown).

$\mathrm{HIP}^{+/+}$mice weighing 20-24 g. Mice were obtained from heterozygous $\times$ heterozygous mating. After fasting for 18 hours with free access to water, the secretagogue caerulein (Sigma Chemical Co., St Louis, Missouri, USA) was administered as seven intraperitoneal injections of $50 \mu \mathrm{g} / \mathrm{kg}$ body weight at hourly intervals, as previously described. ${ }^{25}$ In other experiments, caerulein-treated $\mathrm{PAP} / \mathrm{HIP}^{-1-}$ animals received an intravenous administration of recombinant PAP/HIP (purchased from Dynabio SA, Marseille, France) at time 0 ( $100 \mu \mathrm{g} / \mathrm{kg}$ body weight). All studies were performed in accordance with the European Union regulations for animal experiments.

\section{Preparation of plasma and tissue samples}

Mice were killed at several time intervals from 4 to 21 hours after the first intraperitoneal injection of caerulein. Whole heparinized blood samples were centrifuged at $4^{\circ} \mathrm{C}$, and plasma was stored at $-80^{\circ} \mathrm{C}$ for further studies. The pancreas was removed on ice, weighed, immediately frozen in liquid nitrogen and stored at $-80^{\circ} \mathrm{C}$.

For myeloperoxidase assay, samples were macerated with $0.5 \%$ hexadecyltrimethylammonium bromide in $50 \mathrm{mmol}$ phosphate buffer $\mathrm{pH}$ 6.0. Homogenates were then disrupted for $30 \mathrm{~s}$ using a Labsonic (B Braun, Melsungen, Germany) sonicator at $20 \%$ power and submitted to three cycles of snap freezing in dry ice and thawing before a final $30 \mathrm{~s}$ sonication. Samples were incubated at $60^{\circ} \mathrm{C}$ for 2 hours and then spun down at $4000 \mathrm{~g}$ for $12 \mathrm{~min}$. Supernatants were collected for myeloperoxidase assay.

\section{Biochemical assays}

Amylase and lipase activities were determined with commercially available assays (Roche Biochemicals, Mannheim, Germany). Myeloperoxidase was measured using 3,3',5,5'tetramethylbenzidine as substrate as previously described. ${ }^{25}$ Enzyme activity was recorded at $630 \mathrm{~nm}$. The assay mixture consisted of $20 \mu \mathrm{l}$ supernatant, $10 \mu \mathrm{l}$ tetramethylbenzidine (final concentration $1.6 \mathrm{mmol}$ ) dissolved in dimethylsulphoxide and $70 \mu \mathrm{l}$ hydrogen peroxide (final concentration $3.0 \mathrm{mmol}$ ) diluted in $80 \mathrm{mmol}$ phosphate buffer $\mathrm{pH} 5.4$.

\section{Quantitation of caerulein-induced injuries}

To evaluate necrosis after caerulein-induced pancreatitis in the pancreas of PAP/HIP-expressing and PAP/HIP-deficient mice, formalin-fixed samples were embedded in paraffin and $5 \mu \mathrm{m}$ sections were stained with hematoxilin and eosin. Samples were coded before light microscopy examination and scored by three experienced morphologists. The intensity of necrosis was scored as the product of lesion severity (on a $0-3$ scale) to their extension (in percentage of surface involved ( $1=0-25 \%$; $2=25-50 \% ; 3=50-75 \% ; 4=75-100 \%$ ) leading to a $0-12$ scale. In a similar way, the intensity of inflammation was scored as the product of the degree of infiltration (on a 0-3 scale) to the extension of infiltration evaluated as above.

\section{Semiquantitative reverse transcriptase-polymerase chain reaction}

Expressions of SOCS3, RegI, RegII, RegIII $\alpha$, PAP/HIP (RegIII $\beta$ ), RegIII $\gamma$, TNF $\alpha$, IL6 and ILl $\beta$ mRNA were monitored by semiquantitative reverse transcriptase-polymerase chain reaction (RT-PCR). Briefly, $1 \mu \mathrm{g}$ total pancreatic RNA, purified as previously described, ${ }^{11}$ was used and the sequence amplified by the Life Technologies One Step RT-PCR System according to the manufacturer's protocol. For SOCS3, the forward primer was 5'-CCTTTGACAAGCGGACTCTC-3' and the reverse primer
A
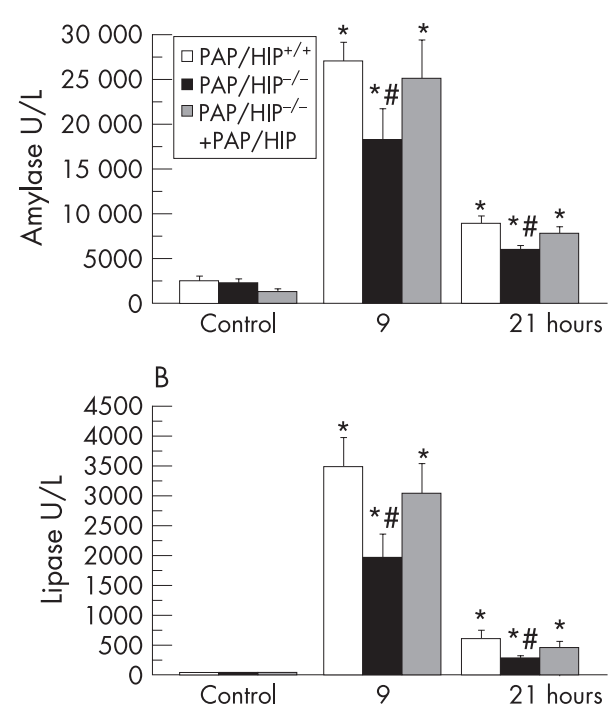
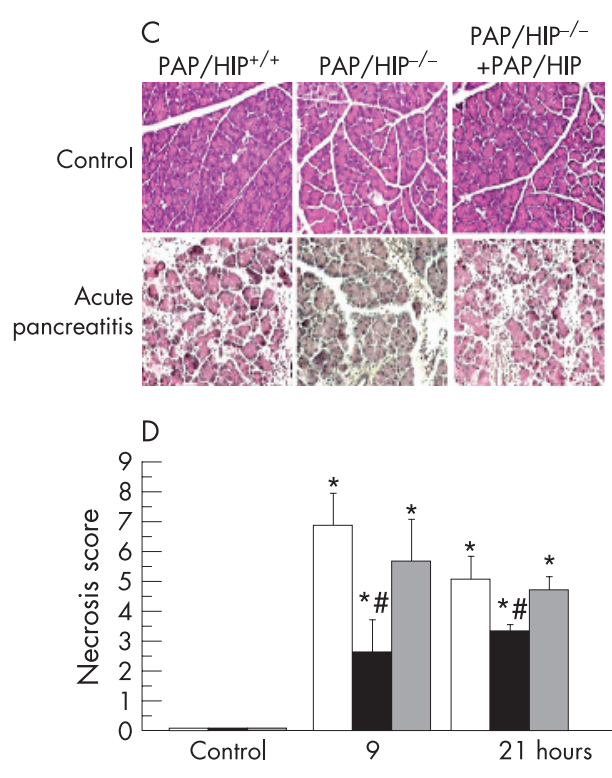

Figure 2 Caerulein-induced pancreatic necrosis is less severe in PAP/HIP ${ }^{-/-}$mice than in wild-type mice. Plasmatic amylase (A) and lipase (B) levels were measured in control PAP/HIP ${ }^{+/+}$, PAP $/ \mathrm{HIP}^{-/-}$and PAP/ $\mathrm{HIP}^{-/-}$treated with recombinant PAP/HIP mice at 9 and 21 hours after the first caerulein injection. (C) Representative images from control pancreas and pancreas after 9 hours of pancreatitis are shown. (D) Necrosis score of histological sections from control pancreas and pancreas after 9 and 21 hours after the induction of acute pancreatitis in PAP/HIP $\mathrm{P}^{++}, \mathrm{PAP} / \mathrm{HIP}^{-/-}$and $\mathrm{PAP} / \mathrm{HIP}^{-/-}$treated with recombinant PAP/ HIP mice. Results are expressed as mean \pm $\mathrm{SE}(\mathrm{n}=8) .{ }^{\#} \mathrm{p}<0.05 \mathrm{PAP} / \mathrm{HIP}^{-/-}$vs PAP/ $\mathrm{HIP}^{+/+}$mice. ${ }^{*} \mathrm{p}<0.05$ vs respective control. 
5' -AGCTCACCAGCCTCATCTGT-3', for RegI the forward primer was 5'-GCCTACAGCTCCTATTGTTAC-3' and the reverse primer was 5'-GGCCATAGGACAGTGAAGC-3', for RegII the forward primer was $5^{\prime}$-CCTGTCATACAGCCAAGGCC-3' and the reverse primer was $5^{\prime}$-CCCAGAGTTCTGCACATCTGTTC, for RegIII $\alpha$ the forward primer was 5'-GCAGTCACCTTTGTCCTGAC-3' and the reverse primer was 5'-CTCCATTGGGTTGTTGACC-3', for PAP/ HIP (RegIII $\beta$ ) the forward primer was $5^{\prime}$-CCTGAAGAATATACCCTCCG-3' and the reverse primer was $5^{\prime}$-CCATGATGCTCTTCAAGACAAATTCG-3', for RegIII $\gamma$ the forward primer was 5'-GGATCTGCAAGACAGACAAGATGC TTCCC-3' and the reverse primer was 5'-GGAGGGAAGGGCCAGAGAAGG-3', for TNF $\alpha$ the forward primer was 5'-AGTCCGGGCAGGTCTACTTT-3' and the reverse primer was 5'-AAGCAAAAGAGGAGGCAACA-3', for IL6 the forward primer was 5'-CCGGAGAGGAGACTTCACAG-3' and the reverse primer was $5^{\prime}$-GGAAATTGGGGTAGGAAGGA-3', for ILl $\beta$ the forward primer was $5^{\prime}$-TCATGGGATGATGATGATAACCTGCT-3' and the reverse primer was 5'-CCCATACTTTAGGAAGACACGGAT-3', and for RL3, a housekeeping gene used as control, the forward primer was 5'-GAAAGAAGTCGTGGAGGCTG-3' and the reverse primer $5^{\prime}$-ATCTCATCCTGCCCAAACAC-3'. RT-PCR products were resolved by $2 \%$ agarose gel electrophoresis and stained with ethidium bromide.

\section{Western blotting}

Antibodies: Rabbit anti-phosphotyrosine-STAT3 $\left(\mathrm{Tyr}^{705}\right)$ antibody was purchased from Cell Signalling Technology (Beverly, Massachusetts, USA). Rabbit antibody against poly(adenosine diphosphate-ribose) polymerase (PARP) was from Calbiochem (Darmstadt, Germany). Antibody against STAT3 was obtained from Santa Cruz Biotechnology (Santa Cruz, California, USA). Monoclonal antibody against $\beta$-actin was purchased from Sigma Chemical Co. (St Louis).

Whole pancreatic extracts: For Western blots, pancreas of mice were rapidly ground in liquid nitrogen. The resulting powder was reconstituted in ice-cold solubilization buffer containing $50 \mathrm{mmol} / \mathrm{l}$ Tris/HCl $(\mathrm{pH} 8), 150 \mathrm{mmol} / \mathrm{l} \mathrm{NaCl}, 1 \%$ Nonidet-P40, $1 \mathrm{mmol} / \mathrm{l}$ phenyl-methylsulphonyl fluoride and a cocktail of protease inhibitors (1/200) (Sigma). Samples were centrifuged at $4^{\circ} \mathrm{C}$ for $10 \mathrm{~min}$ at $10000 \mathrm{~g}$. Supernatants were recovered, and total amounts of protein were determined using the Bradford method.

Nuclear pancreatic extracts: To obtain nuclear extracts, pancreas was lysed using the nuclear extract kit from Active Motif (Carlsbad, California, USA) following the manufacturer's protocol. Total amounts of protein were determined as described above.

Sodium dodecylsulphate-polyacrylamide gel electrophoresis and Western blotting: Fifty micrograms of protein was submitted to $12 \%$ sodium dodecylsulphate-polyacrylamide gel electrophoresis then blotted following standard methods. Migration was calibrated using Bio-Rad standard proteins (Hercules, California, USA) with markers covering a 7-240 kDa range. Non-specific binding to the membrane was blocked by $5 \%$ bovine serum albumin in Tris-buffered saline (TBS) for 1 hour at $4^{\circ} \mathrm{C}$. Blots were incubated overnight at $4{ }^{\circ} \mathrm{C}$ with rabbit anti-STAT3 antibody (1:500), anti-PARP antibody (1:500), a rabbit polyclonal antibody specific to phosphotyrosine-STAT3 (1:1000) or a monoclonal anti $\beta$-actin (1:1500) diluted in 5\% bovine serum albumin. Membranes were this washed with TBS-0.1\% Triton and incubated with a secondary goat-antirabbit-horseradish peroxidase or goat-anti-mouse-horseradish peroxidase antibody (1:3000) obtained from Santa Cruz Biotechnology (Santa Cruz, California, USA) diluted in 5\% dry non-fat milk in TBS for 1 hour at room temperature. Finally, membranes were washed with TBS- $0.1 \%$ Triton, developed with the ECL-detection system (Santa Cruz Biotechnology), quickly dried and exposed to ECL film.

\section{Immunohistochemistry}

Paraffin-embedded pancreas samples were deparaffinized and after blocking non-specific binding sites, sections were incubated with anti-active caspase 3 antibody (1:500; Promega, Madison, Wisconsin, USA). The sections were then incubated with a biotinylated-linked antibody for $20 \mathrm{~min}$ and with peroxidase-labeled streptavidin at room temperature for $20 \mathrm{~min}$. The chromogen used for the colour reaction was 3amino-9-ethyl-carbazole. The areas labelled by caspase 3 immunoreactivity were quantified using an Olympus BX61 automated microscope $(10 \times$ objective) using a Samba 2050 image analyser (Samba Technologies, Meylan, France).

\section{Densitometric analysis}

ImageJ 1.32 software from http://rsbweb.nih.gov/ij/download.html was used to quantify intensities of the bands obtained in Western blots and RT-PCR experiments.

\section{Statistical analysis}

Data shown in the figures indicate the means \pm SE. Differences between groups were compared using the non-parametric Mann-Whitney $U$ test. Asterisks in the figures indicate statistically significant differences $(p<0.05)$.

\section{RESULTS}

\section{Expression of Regl, Regll, Reglll $\alpha$ and Reglll $\gamma$ in PAP/ HIP $^{-/}$mice}

Before comparing $\mathrm{PAP} / \mathrm{HIP}^{-/-}$and $\mathrm{PAP} / \mathrm{HIP}^{+/+}$mice during pancreatitis, we checked that their pancreatic weight as well as amylase, lipase and trypsinogen pancreatic contents were similar (not shown) and that during pancreatitis PAP/HIP was indeed expressed in wild-type and not in $\mathrm{PAP} / \mathrm{HIP}^{-1-}$ mice (fig 1A). Also, because PAP/HIP (RegIII $\beta$ ) belongs to a family of five proteins whose functions could be similar, it was conceivable that the suppression of PAP/HIP expression induces, by a compensatory mechanism, the overexpression of other members. This had to be checked because such compensation should be taken into account when analyzing results involving PAP/HIP ${ }^{-1-}$ mice. We assessed the expression of RegI, RegII, RegIII $\alpha$ and RegIII $\gamma$, in the pancreas of PAP/ $\mathrm{HIP}^{-/-}$and PAP/HIP ${ }^{+/+}$mice, after treatment with caerulein. As shown in figure 1B, no upregulation was observed in RegI, RegII and RegIII $\alpha$ expressions, which were, on the contrary, slightly diminished. As expected, PAP/HIP (RegIII $\beta$ ) was not detected, nor RegIII $\gamma$, whose expression does not occur in the pancreas. $^{12}$

\section{Caerulein-induced pancreatic necrosis is more severe in wild-type than in PAP/HIP-deficient mice}

As previously described, ${ }^{28}$ wild-type mice given intraperitoneal injections of the secretagogue caerulein at a supramaximal dose develop acute necrotizing pancreatitis. We compared the severities of caerulein-induced pancreatitis in wild-type and $\mathrm{PAP} / \mathrm{HIP}^{-/-}$mice by measuring amylase and lipase levels in serum and by assessing the extent of acinar cell necrosis. As shown in figure 2, the induction of pancreatitis was followed by a time-dependent increase in serum amylase and lipase levels. Amylasemia and lipasemia were, however, significantly higher in wild-type than in $\mathrm{PAP} / \mathrm{HIP}^{-/-}$mice, by $33 \%$ and $43 \%$, respectively, suggesting that pancreatitis was more severe in wild-type mice (fig 2). In the PAP/HIP ${ }^{-1-}$ mice treated with recombinant PAP/HIP, serum levels of amylase and lipase were similar to those of wild-type mice. Indeed, histological examination of pancreatic sections revealed that necrotic areas 
A
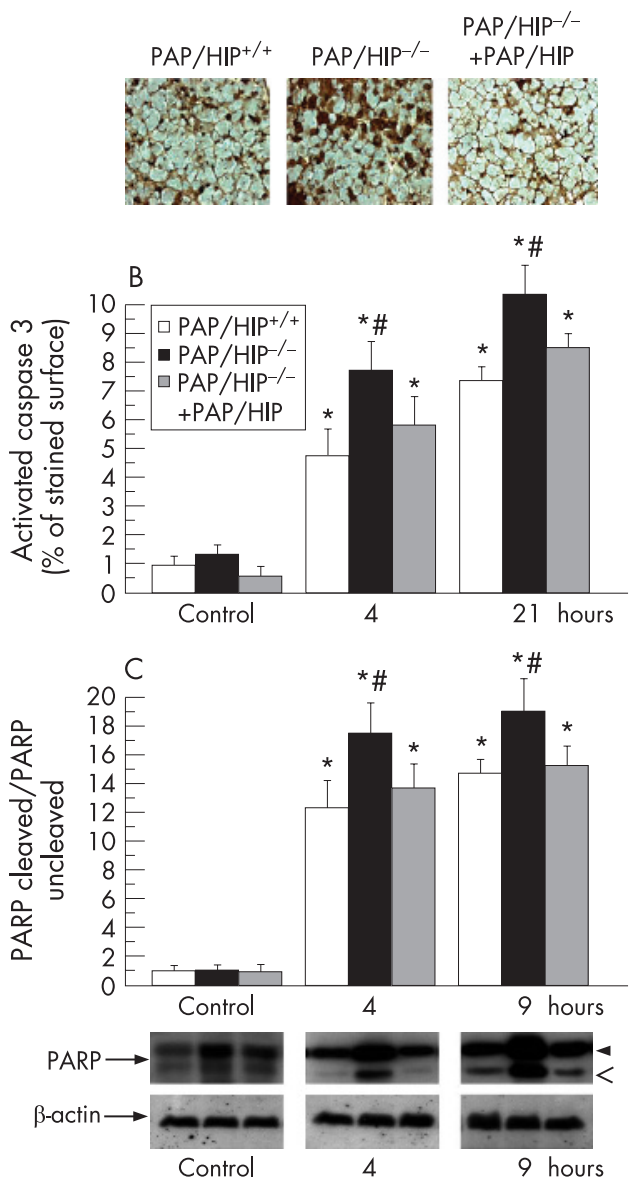

Figure 3 Caerulein-induced pancreatic induces more apoptosis in PAP/ $\mathrm{HIP}^{-/-}$than in wild-type mice. (A) Immunohistochemistry of active caspase 3 on pancreatic paraffin sections from $\mathrm{PAP} / \mathrm{HIP}^{+/+}, \mathrm{PAP} / \mathrm{HIP}^{-/-}$and recombinant PAP/HIP-treated PAP/HIP ${ }^{-/-}$mice after 4 and 9 hours of pancreatic induction. Representative images from PAP/HIP ${ }^{+/+}, \mathrm{PAP} / \mathrm{HIP}^{-/}$ and recombinant PAP/HIP-treated PAP/HIP ${ }^{-1-}$ mice 4 hours after the first caerulein injection are shown. (B) The graph shows the percentage of surface with active caspase 3 -positive staining. No staining was observed in the absence of primary antibody (data not shown). (C) Assessment of poly(adenosine diphosphate-ribose) polymerase (PARP) cleavage by Western blot analysis in pancreatic homogenates from PAP/HIP ${ }^{+/+}$, PAP/ $\mathrm{HIP}^{-/-}$and PAP/HIP ${ }^{-/-}$treated with recombinant PAP/HIP mice at 4 and 9 hours after caerulein injection. Upper band corresponds to uncleaved PARP (1 $16 \mathrm{kDa}$ ) and lower band to the cleaved fragment (85 kDa). $\beta$-Actin was used as loading control. The intensity of bands was measured and the represented values correspond to the (PARP cleaved/PARP uncleaved)/ $\beta$ actin ratio. Representative images of the results are shown (lower panel). Results are expressed as mean $+\mathrm{SE}(\mathrm{n}=8)$. ${ }^{*} \mathrm{p}<0.05 \mathrm{PAP} / \mathrm{HIP}^{-1-}$ vs $\mathrm{PAP} / \mathrm{HIP}^{+/+}$mice. ${ }^{*} \mathrm{p}<0.05$ vs respective control.

were more extended in wild-type than in $\mathrm{PAP} / \mathrm{HIP}^{-/-}$mice (fig 2).

\section{Pancreatic apoptosis in PAP/HIP-deficient and wild- type mice during caerulein-induced pancreatitis} We compared the extent of pancreatic apoptosis in PAP/HIP ${ }^{-/-}$ and wild-type mice after the induction of pancreatitis by monitoring caspase 3 activity and PARP cleavage in pancreatic tissue. The percentage of cells showing caspase 3 activity was estimated by quantitative immunohistochemistry and the extent of PARP cleavage in tissue was assessed by Western blot. Significantly larger caspase 3-labeled areas were observed in $\mathrm{PAP} / \mathrm{HIP}^{-/-}$mouse pancreas, compared with wild-type controls, revealing more abundant apoptosis in $\mathrm{PAP} / \mathrm{HIP}^{-/-}$ animals (fig 3). These areas were smaller when recombinant PAP/HIP was administered to PAP/HIP ${ }^{-/-}$mice before the induction of acute pancreatitis (fig 3 ). These findings were confirmed by the higher level of caspase-specific PARP cleavage observed in $\mathrm{PAP} / \mathrm{HIP}^{-/-}$mouse pancreas compared with wildtype (fig 3). Again, that difference was almost completely abolished when PAP/HIP ${ }^{-/-}$mice were treated with recombinant PAP/HIP before starting pancreatitis induction (fig 3). These results indicate that, during caerulein-induced pancreatitis, the pancreas is more sensitive to apoptosis in PAP/HIP ${ }^{-/-}$ than in wild-type mice, in agreement with the anti-apoptotic effect attributed to PAP/HIP. ${ }^{15}$ 20-22

During experimental acute pancreatitis, the pancreatic inflammatory response is more severe in PAP/HIPdeficient than in wild-type mice

Contrary to apoptosis, the necrosis of pancreatic cells generates inflammation, as a result of the release of cell debris. Such inflammation is evidenced by polymorphonuclear (PMN) leukocyte accumulation around the areas of tissue damage. To compare the extent of inflammation in $\mathrm{PAP} / \mathrm{HIP}^{-/-}$and wild-type mice upon the induction of pancreatitis, we assessed PMN leukocyte infiltration in the pancreas by monitoring the activity in tissue of myeloperoxidase, an enzyme specifically found in PMN leukocytes. On that basis, PMN leukocyte sequestration appeared significantly higher in $\mathrm{PAP} / \mathrm{HIP}^{-/-}$than in wild-type mice, but the increase was significantly reduced when mice were treated with recombinant PAP/HIP before the induction of pancreatitis (fig 4). Assessment of inflammation by quantitative histological analysis led to similar conclusions. During the course of pancreatitis, the inflammatory infiltrate (mainly neutrophils) was significantly more abundant in the pancreas of PAP/HIP ${ }^{-/-}$than in wild-type mice and, again, that increase was more limited when PAP/HIP was administered before inducing pancreatitis (fig 4). Finally, expression of the proinflammatory cytokines TNF $\alpha$, IL6 and ILI $\beta$ mRNAs were significantly higher in $\mathrm{PAP} / \mathrm{HIP}^{-/-}$mice compared with $\mathrm{PAP} / \mathrm{HIP}^{+/}$ ${ }^{+}$mice, and the expression of these cytokines was decreased after treating PAP/HIP ${ }^{-/-}$mice with recombinant PAP/HIP (fig 4). These results are, however, in agreement with the anti-inflammatory function previously reported for PAP/HIP. ${ }^{17} 2425$

\section{STAT3 activity and SOCS3 expression in the pancreas from PAP/HIP ${ }^{-1-}$ mice}

We compared wild-type and $\mathrm{PAP} / \mathrm{HIP}^{-1}$ mice to assess in vivo Jak/STAT3/SOCS3 activation by PAP/HIP. STAT3 is transiently activated in the pancreas with acute pancreatitis, as evidenced by Western blot of nuclear extracts from pancreatic cells (fig. 5). Interestingly, the phosphorylated (activated) STAT3 level was significantly higher in wild-type than in $\mathrm{PAP} / \mathrm{HIP}^{-/-}$mice (fig 5). Moreover, figure 5 shows that the induction of pancreatitis decreases the total STAT3 level in the pancreas of wild-type mice. A smaller decrease was seen in the pancreas of $\mathrm{PAP} / \mathrm{HIP}^{-1-}$ mice. As a control, we monitored in the pancreas of these animals the concentration of SOCS3 mRNA, a STAT3specific target, which was actually found to be significantly higher in wild-type than in PAP/HIP ${ }^{-/-}$mice (fig 6). In PAP/ $\mathrm{HIP}^{-/-}$mice treated with recombinant PAP/HIP, STAT3 activation (fig 5) as well as SOCS3 mRNA expression (fig 6) reached values close to those found in wild-type pancreas. These results support a role of PAP/HIP in activating the Jak/ STAT3/SOCS3 anti-inflammatory pathway in vivo, and confirm the results of our previous in-vitro studies. ${ }^{17}$

\section{DISCUSSION}

$\mathrm{PAP} / \mathrm{HIP}$ was first identified as an additional secretory protein, appearing in rat pancreatic juice after the induction of 

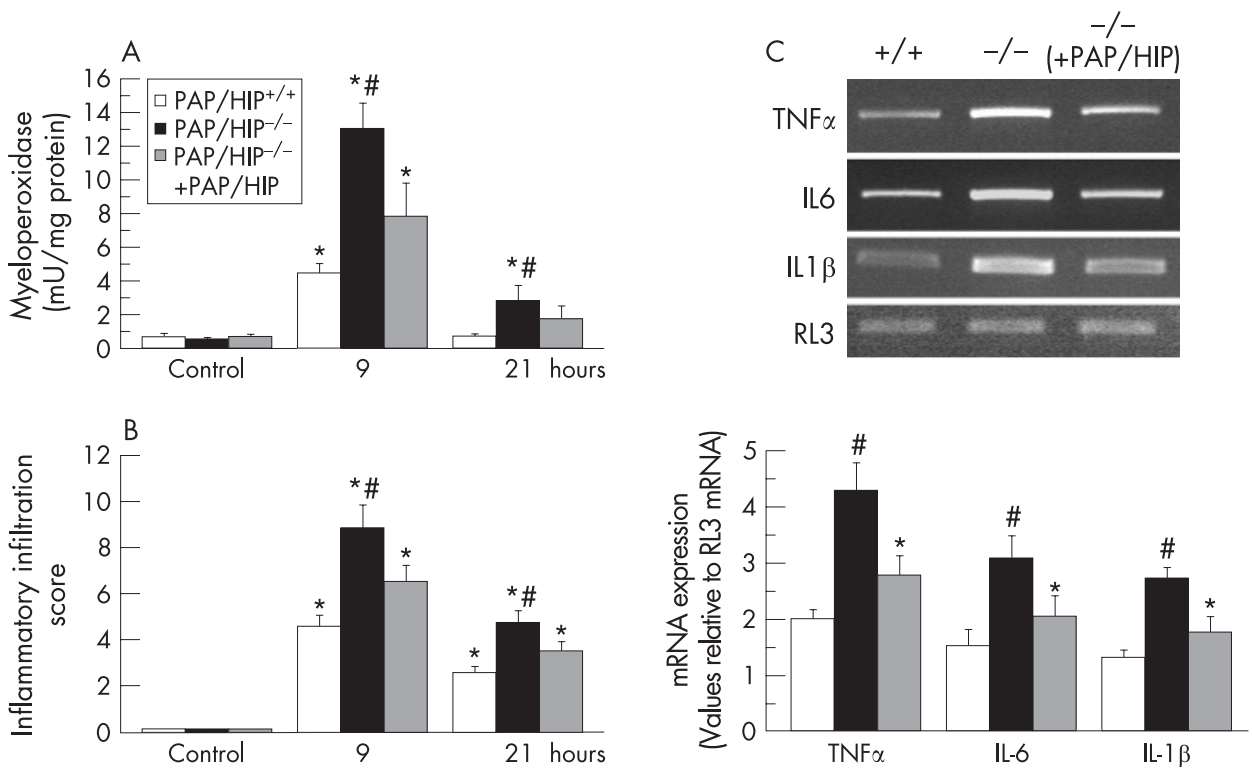

Figure 4 Caerulein-induced pancreatic inflammation is more severe in PAP/HIP ${ }^{-/-}$than in wild-type mice. (A) Pancreatic myeloperoxidase activity was measured in the pancreas of control PAP/HIP ${ }^{+/}, \mathrm{PAP} / \mathrm{HIP}^{-/}$and recombinant PAP/HIP-treated PAP/HIP ${ }^{-}$mice 9 and 21 hours after caerulein injection. (B) The inflammatory cell infiltration score was quantified on histological sections from pancreas 0 (control), 9 and 21 hours after the induction of acute pancreatitis in PAP/HIP ${ }^{/++}, \mathrm{PAP} / \mathrm{HIP}^{-/-}$and recombinant PAP/HIP-treated PAP/HIP ${ }^{-/-}$mice. Results are expressed as mean $\pm \mathrm{SE}(\mathrm{n}=8)$. ${ }^{\#} \mathrm{p}<0.05 \mathrm{PAP} / \mathrm{HIP}^{-/-}$vs PAP/HIP ${ }^{+/+}$mice. ${ }^{*} \mathrm{p}<0.05$ vs respective control. (C) Expression of tumour necrosis factor $\alpha$, interleukin (IL) 6 and IL1 $\beta$ mRNA was assessed by semiquantitative reverse transcriptase-polymerase chain reaction analysis in pancreatic RNA from PAP/HIP ${ }^{+/+}, \mathrm{PAP} / \mathrm{HIP} \mathrm{P}^{-1-}$ mice and recombinant PAP/HIP-treated PAP/HIP ${ }^{-1-}$ mice, 9 hours after caerulein treatment. RL3 mRNA was used as a housekeeping control. The intensity of bands was measured and the represented values correspond to the cytokine mRNA/RL3 mRNA ratio. Images from one representative mouse for each group are shown (upper panel). Results are expressed as mean $\pm \mathrm{SE}(\mathrm{n}=5)$. ${ }^{\#} \mathrm{p}<0.05 \mathrm{PAP} / \mathrm{HIP}^{-/-} \mathrm{vs} \mathrm{PAP} / \mathrm{HIP}^{+/+}$mice. ${ }^{*} \mathrm{p}<0.05$ vs respective control.

experimental pancreatitis. ${ }^{1}$ It belongs to a five-member family of secretory proteins containing a C-type lectin-like domain linked to a short $\mathrm{N}$-terminal peptide. ${ }^{29}$ Besides the exocrine pancreas, PAP/HIP is expressed in several other organs such as the endocrine pancreas, the intestine, the pituitary, the uterus and the motoneuron, either constitutively or after induction by a stress. PAP/HIP can also appear in tumours from tissues such as the colon and liver in which the protein is not expressed in normal conditions. ${ }^{8}{ }^{10}$ Several functions have been suggested for PAP/HIP, some of them being apparently unrelated. ${ }^{29}$ What makes the protein unique and especially interesting, however, is that it can be mitogenic, ${ }^{6} 1920$ anti-apoptotic ${ }^{15}{ }^{20-22}$ and antiinflammatory, ${ }^{74-26}$ suggesting that PAP/HIP is a key regulatory factor. It is noteworthy that other members of the PAP/HIP family may have different functions. For example, the RegIII $\gamma$ isoform, which is expressed in Paneth cells but not in the pancreas, is bactericidal and may participate in the control of intestinal flora. ${ }^{30}$ Because data on the function of PAP/HIP were obtained on different tissues using various experimental models, in vivo or in vitro, their integration into a general scheme of PAP/HIP function remains difficult. To gain further insight into this problem we used a PAP/HIP ${ }^{-/-}$mouse model
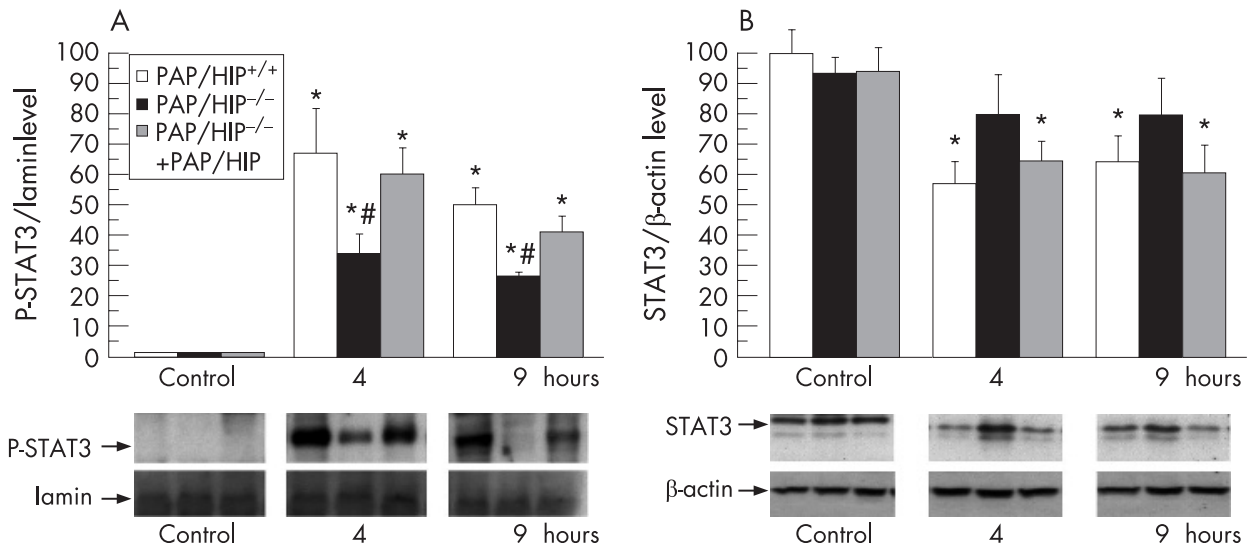

Figure 5 STAT3 activation in PAP/HIP $\mathrm{P}^{+/+}$and PAP/HIP ${ }^{-/-}$mouse pancreas during caerulein-induced acute pancreatitis. (A) Assessment of Tyr ${ }^{705}$. phosphorylated STAT3 levels by Western blot analysis in nuclear pancreatic homogenates from PAP/HIP ${ }^{+/+}$, PAP/HIP ${ }^{-1-}$ and recombinant PAP/HIPtreated PAP/HIP ${ }^{-1}$ mice, 4 and 9 hours after caerulein injection. Lamin $b$ was used as nuclear extracts loading control. The intensity of bands was measured and the represented values correspond to the STAT3-P/lamin b ratio. (B) Assessment of total STAT3 levels by Western blot analysis in pancreatic homogenates from PAP/HIP ${ }^{+/+}, \mathrm{PAP} / \mathrm{HIP}^{-/-}$and recombinant PAP/HIP-treated PAP/HIP ${ }^{-/-}$mice, 4 and 9 hours after caerulein injection. $\beta$-Actin was used as loading control. The intensity of bands was measured and the represented values correspond to the STAT3/ $\beta$-actin ratio. Representative images of the results are shown in each case (lower panels). Results are expressed as mean $\pm \mathrm{SE}(\mathrm{n}=8)$. ${ }^{\#} \mathrm{p}<0.05 \mathrm{PAP} / \mathrm{HIP}{ }^{-/-}$vs PAP/HIP ${ }^{+/+}$mice. ${ }^{*} \mathrm{p}<0.05 \mathrm{vs}$ respective control. 


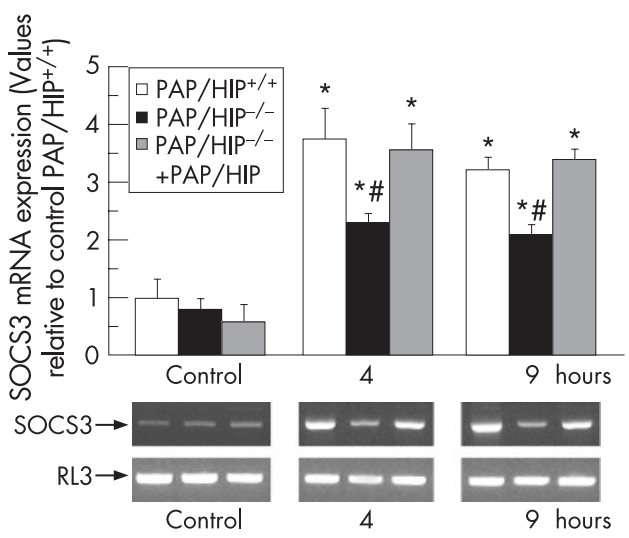

Figure $6 \mathrm{SOCS} 3$ expression in $\mathrm{PAP} / \mathrm{HIP}^{+/+}$and $\mathrm{PAP} / \mathrm{HIP}^{-/-}$mouse pancreas during caerulein-induced acute pancreatitis. Assessment of SOCS3 mRNA levels by semiquantitative reverse transcriptase-polymerase chain reaction analysis in pancreatic RNA from PAP/HIP $\mathrm{P}^{+/+}, \mathrm{PAP} / \mathrm{HIP}^{-/-}$ and recombinant PAP/HIP-treated PAP/HIP ${ }^{-1-}$ mice, 4 and 9 hours after caerulein treatment. RL3 mRNA was used as a housekeeping control. The intensity of bands was measured and the represented values correspond to the ratio SOCS3/RL3. Representative images of the results are shown (lower panels). Results are expressed as mean \pm SE $(n=8)$. ${ }^{\#} p<0.05$ $\mathrm{PAP} / \mathrm{HIP}^{-/-}$vs PAP/HIP ${ }^{+/+}$mice. ${ }^{*} \mathrm{p}<0.05$ vs respective control.

to assess in vivo the consequences of PAP/HIP deletion on the pancreas during acute pancreatitis.

Induction by supramaximal doses of caerulein was chosen as model of pancreatitis because it is easy to implement, reproducible with homogeneous distribution of the necrotic lesions in the pancreas, and is not lethal. In this model we found that pancreatic necrosis was less severe in $\mathrm{PAP} / \mathrm{HIP}^{-1-}$ than in wild-type mice. Amylase and lipase serum levels, two specific markers of severity, were lower in PAP/HIP ${ }^{-1-}$ animals, and histological examination revealed that necrotic areas were more extensive in wild-type mice. These results strongly suggest that pancreatic cells are less sensitive to necrosis in $\mathrm{PAP} / \mathrm{HIP}^{-1-}$ than in wild-type mice. In addition, treatment of $\mathrm{PAP} / \mathrm{HIP}^{-1-}$ mice with recombinant $\mathrm{PAP} / \mathrm{HIP}$ was able to reverse this effect, as shown in figure 2. During acute pancreatitis, acinar cell death occurs by necrosis or by apoptosis. ${ }^{31}$ Large areas of necrosis are found in severe acute pancreatitis, whereas mild forms of the disease are associated with abundant apoptotic cells and reduced areas of necrosis, if any. It is generally accepted that when a tissue is submitted to aggression, apoptosis is triggered to prevent cells from undergoing necrosis, a "clean" death avoiding the massive release of cell debris that might generate a necrosis-inflammationnecrosis vicious circle. ${ }^{32}{ }^{33}$ If the aggression is sufficiently strong, however, as in our model of caerulein-induced pancreatitis when used in wild-type animals, necrosis occurs before apoptosis takes place. The induction of apoptosis might therefore reduce the severity of experimental pancreatitis. This was indeed shown in a mouse model of caerulein-induced acute pancreatitis ${ }^{32}$ and confirmed in a rat model of caeruleininduced pancreatitis when apoptosis was induced by an extract of Artemisia asiatica. ${ }^{34}$ On the contrary, inhibition of apoptosis worsens the disease. For example, mice deficient in the Cx32 gene, which are resistant to crambene-induced apoptosis, are more susceptible to acute pancreatitis than their wild-type counterparts. ${ }^{35}$ Therefore, one explanation for the lower level of necrosis observed in $\mathrm{PAP} / \mathrm{HIP}^{-1-}$ mice could be that the absence of PAP/HIP anti-apoptotic activity favours apoptosis. As a control, we monitored two markers of apoptosis, caspasedependent PARP cleavage and caspase 3 activation, in the pancreas of $\mathrm{PAP} / \mathrm{HIP}^{-/-}$and wild-type animals during pancreatitis. Both were significantly higher in $\mathrm{PAP} / \mathrm{HIP}^{-/-}$mice, confirming that the absence of PAP/HIP favours apoptosis, in agreement with the anti-apoptotic role of PAP/HIP previously demonstrated in vitro. ${ }^{15}{ }^{20-22}$ Further confirmation was obtained by showing that the administration of recombinant PAP/HIP to $\mathrm{PAP} / \mathrm{HIP}^{-1-}$ mice partly restored an anti-apoptotic activity, as judged by decreased caspase 3 activation and PARP cleavage.

Pancreatic necrosis generates an inflammatory response during acute pancreatitis whose intensity correlates with the severity of pancreatitis. Because necrosis was significantly less abundant in PAP/HIP ${ }^{-/-}$animals, we expected that they would also show less inflammation. This was not the case, as PMN leukocyte accumulation and pro-inflammatory cytokine expression were increased in pancreas from $\mathrm{PAP} / \mathrm{HIP}^{-/-}$mice. Again, the administration of recombinant $\mathrm{PAP} / \mathrm{HIP}$ to $\mathrm{PAP} / \mathrm{HIP}^{-/-}$ mice could reverse the phenomenon, corroborating the antiinflammatory role of PAP/HIP in vivo, in agreement with invitro data. ${ }^{7}{ }^{17} 25$ In addition, the intensity of inflammation in the absence of PAP/HIP, although necrosis was limited, suggests that in physiological conditions the anti-inflammatory activity of PAP/HIP is quite strong.

In-vitro studies ${ }^{17}$ demonstrated that the anti-inflammatory function of PAP/HIP involves the Jak/STAT3/SOCS3 pathway. STAT3 is a cytoplasmic factor that translocates into the nucleus after its phosphorylation by the Jak kinase. Once in the nucleus, the activated STAT3 triggers the transcription of several genes including SOCS3, which in turn inhibits the pro-inflammatory cytokine cascade. To evaluate the situation in vivo, we monitored STAT3 activity and SOCS3 concentrations in the pancreas of wild-type and PAP/HIP ${ }^{-1-}$ mice. The level of the activated (phosphorylated) form of STAT3 and the concentration of SOCS3 mRNA were indeed significantly lower in PAP/ $\mathrm{HIP}^{-/-}$animals, consistent with the absence of PAP/HIP in pancreatic cells. That some STAT3 activity remains in PAP/ $\mathrm{HIP}^{-1-}$ animals is not surprising because it can also be activated by other cytokines, ${ }^{36}$ growth factors ${ }^{37}$ or various stress agents. $^{38}$

Altogether, these results show that the anti-apoptotic and anti-inflammatory functions described in vitro for PAP/HIP are also observed in vivo. Therefore, PAP/HIP appears to be a key factor in the pancreatic response to acute pancreatitis injury.

\section{ACKNOWLEDGEMENTS}

This work was supported in part by grant FIS PI050599 from the Instituto de Salud Carlos III and from INSERM. MG is the recipient of a post-vert (INSERM) fellowship and EF-P is the recipient of a Ramón y Cajal research contract.

\section{Authors' affiliations \\ Meritxell Gironella, Aude LeGoffic, Stéphane Garcia, Jean-Charles Dagorn, Juan L lovanna, INSERM, U.624, F-13009 Marseille, France Emma Folch-Puy, Daniel Closa, Department of Experimental Pathology, IIBB-CSIC, IDIBAPS, CIBEREHD Barcelona, Spain \\ Laurence Christa, Laboratoire de Biochimie Métabolique, Hôpital Necker Enfants-Malades, Paris, France \\ Andrew Smith, Luis Tebar, Stephen P Hunt, Department of Anatomy and Developmental Biology, University College London, London, UK \\ Rosemary Bayne, Andrew J H Smith, Gene Targeting Laboratory, Institute for Stem Cell Research, University of Edinburgh, The King's Buildings, Edinburgh, UK \\ Conflict of interest: None declared. \\ REFERENCES \\ 1 Keim V, Rohr G, Stockert HG, et al. An additional secretory protein in the rat pancreas. Digestion 1984;29:242-249. \\ 2 Keim V, lovanna JL, Rohr G, et al. Characterization of a rat pancreatic secretory protein associated with pancreatitis. Gastroenterology 1991;100:775-782. \\ 3 lovanna JL, Keim V, Bosshard A, et al. PAP, a pancreatic secretory protein induced during acute pancreatitis, is expressed in rat intestine. Am J Physiol 1993;265:G611-G618.}


4 Katsumata N, Chakraborty C, Myal Y, et al. Molecular cloning and expression of peptide 23, a growth hormone-releasing hormone-inducible pituitary protein. Endocrinology 1995; 136:1332-1339.

5 Chakraborty C, Vrontakis M, Molnar P, et al. Expression of pituitary peptide 23 in the rat uterus: regulation by estradiol. Mol Cell Endocrinol 1995;108:149-154.

6 Livesey FJ, O'Brien JA, Li M, et al. A Schwann cell mitogen accompanying regeneration of motor neurons. Nature 1997;390:614-618.

7 Gironella M, lovanna JL, Sans $M$, et al. Anti-inflammatory effects of pancreatitis associated protein in inflammatory bowel disease. Gut 2005;54:1244-1253.

8 Rechreche H, Montalto G, Mallo GV, et al. Pap, reg I alpha and reg I beta mRNAs are concomitantly up-regulated during human colorectal carcinogenesis. Int J Cancer 1999;81:688-694.

9 Christa L, Simon MT, Brezault-Bonnet C, et al. Hepatocarcinoma-intestinepancreas/pancreatic associated protein (HIP/PAP) is expressed and secreted by proliferating ductules as well as by hepatocarcinoma and cholangiocarcinoma cells. Am J Pathol 1999; 155:1525-1533.

10 Lasserre C, Christa L, Simon MT, et al. A novel gene (HIP) activated in human primary liver cancer. Cancer Res 1992;52:5089-5095.

11 lovanna J, Orelle B, Keim V, et al. Messenger RNA sequence and expression of rat pancreatitis-associated protein, a lectin-related protein overexpressed during acute experimental pancreatitis. J Biol Chem 1991;266:24664-24669.

12 Narushima Y, Michiaki U, Nakagawara K, et al. Structure, chromosomal localization and expression of mouse genes encoding type III Reg, Reglll $\alpha$, Reglll $\beta$, Reglll $\gamma$. Gene 1997;185:159-168.

13 Orelle B, Keim V, Masciotra L, et al. Human pancreatitis-associated protein. Messenger RNA cloning and expression in pancreatic diseases. J Clin Invest 1992:90:2284-2291.

14 Dusetti NJ, Ortiz EM, Mallo GV, et al. Pancreatitis-associated protein I (PAP I), an acute phase protein induced by cytokines. Identification of two functional interleukin-6 response elements in the rat PAP I promoter region. J Biol Chem 1995:270:22417-22421.

15 Ortiz EM, Dusetti NJ, Vasseur S, et al. The pancreatitis-associated protein is induced by free radicals in AR4-2J cells and confers cell resistance to apoptosis. Gastroenterology 1998;114:808-816.

16 Vaccaro MI, Calvo EL, Suburo AM, et al. Lipopolysaccharide directly affects pancreatic acinar cells: implications on acute pancreatitis pathophysiology. Dig Dis Sci 2000:45:915-926.

17 Folch-Puy E, Granell S, Dagorn JC, et al. Pancreatitis-associated protein I suppresses NF-kappa B activation through a JAK/STAT-mediated mechanism in epithelial cells. J Immunol 2006;176:3774-3779.

18 Aggarwal S, Xie MH, Maruoka M, et al. Acinar cells of the pancreas are a target of interleukin-22. J Interferon Cytokine Res 2001;21:1047-1053.

19 Moucadel V, Soubeyran P, Vasseur S, et al. Cdx1 promotes cellular growth of epithelial intestinal cells through induction of the secretory protein PAP I. Eur J Cell Biol 2001;80:156-163.

20 Simon MT, Pauloin A, Normand G, et al. HIP/PAP stimulates liver regeneration after partial hepatectomy and combines mitogenic and anti-apoptotic functions through the PKA signaling pathway. FASEB J 2003;17:1441-1450.
21 Malka D, Vasseur S, Bodeker H, et al. Tumor necrosis factor alpha triggers antiapoptotic mechanisms in rat pancreatic cells through pancreatitis-associated protein I activation. Gastroenterology 2000;1 19:816-828.

22 Nishimune H, Vasseur S, Wiese S, et al. Reg-2 is a motoneuron neurotrophic factor and a signalling intermediate in the CNTF survival pathway. Nat Cell Biol 2000;2:906-914

23 Lieu HT, Batteux F, Simon MT, et al. HIP/PAP accelerates liver regeneration and protects against acetaminophen injury in mice. Hepatology 2005:42:618-626.

24 Heller A, Fiedler F, Schmeck J, et al. Pancreatitis-associated protein protects the lung from leukocyte-induced injury. Anesthesiology 1999;91:1408-1414.

25 Vasseur S, Folch-Puy E, Hlouschek V, et al. P8 improves pancreatic response to acute pancreatitis by enhancing the expression of the anti-inflammatory protein pancreatitis-associated protein I. J Biol Chem 2003;279:7199-7207.

26 Zhang H, Kandil E, Lin YY, et al. Targeted inhibition of gene expression of pancreatitis-associated proteins exacerbates the severity of acute pancreatitis in rats. Scand J Gastroenterol 2004;39:870-881

27 Lieu HT, Simon MT, Nguyen-Khoa T, et al. Reg2 inactivation increases sensitivity to Fas hepatotoxicity and delays liver regeneration post-hepatectomy in mice. Hepatology 2006;44:1452-1464.

28 Willemer S, Elsasser HP, Adler G. Hormone-induced pancreatitis. Eur Surg Res 1992;24:29-39.

29 lovanna JL, Dagorn JC. The multifunctional family of secreted proteins containing a C-type lectin-like domain linked to a short $\mathrm{N}$-terminal peptide. Biochim Biophys Acta 2005;1723:8-18.

30 Cash HL, Whitham CV, Behrendt CL, et al. Symbiotic bacteria direct expression of an intestinal bactericidal lectin. Science 2006;313(5790): 1126-1130.

31 Kaiser AM, Saluja AK, Sengupta A, et al. Relationship between severity, necrosis, and apoptosis in five models of experimental acute pancreatitis. Am J Physiol 1995;269:C1295-C1304.

32 Bhatia M, Wallig MA, Hofbaver B, et al. Induction of apoptosis in pancreatic acinar cells reduces the severity of acute pancreatitis. Biochem Biophys Res Commun 1998;246:476-483.

33 Mareninova OA, Sung KF, Hong $P$, et al. Cell death in pancreatitis: caspases protect from necrotizing pancreatitis. J Biol Chem 2006;281:3370-3381.

34 Hahm KB, Kim JH, You BM, et al. Induction of apoptosis with an extract of Artemisia asiatica attenuates the severity of cerulein-induced pancreatitis in rats. Pancreas 1998;17:153-157.

35 Frossard JL, Rubbia-Brandt L, Wallig MA, et al. Severe acute pancreatitis and reduced acinar cell apoptosis in the exocrine pancreas of mice deficient for the Cx32 gene. Gastroenterology 2003;124:481-493.

36 Hebenstreit D, Horejs-Hoeck J, Duschl A. JAK/STAT-dependent gene regulation by cytokines. Drug News Perspect 2005;18:243-249.

37 Calo V, Migliavacca M, Bazan V, et al. STAT proteins: from normal control of cellular events to tumorigenesis. J Cell Physiol 2003;197:157-168.

38 Yu HM, Zhi JL, Cui Y, et al. Role of the JAK-STAT pathway in protection of hydrogen peroxide preconditioning against apoptosis induced by oxidative stress in PC12 cells. Apoptosis 2006;11:931-941.

\section{Let us assist you in teaching the next generation}

Figures from all articles on our website can be downloaded as a PowerPoint slide. This feature is ideal for teaching and saves you valuable time. Just click on the image you need and choose the "PowerPoint Slide for Teaching" option. Save the slide to your hard drive and it is ready to go. This innovative function is an important aid to any clinician, and is completely free to subscribers. (Usual copyright conditions apply.) 